Revue des patrimoines

\title{
Le programme ARTEMIS : nouvel outil pour la datation radiocarbone AMS (Spectromètre de Masse par Accélérateur) et nouvelles problématiques
}

\section{Cyrille Billard}

\section{(2) OpenEdition \\ Journals}

Édition électronique

URL : http://journals.openedition.org/insitu/3342

DOI : $10.4000 /$ insitu.3342

ISSN : $1630-7305$

Éditeur

Ministère de la culture

Référence électronique

Cyrille Billard, «Le programme ARTEMIS : nouvel outil pour la datation radiocarbone AMS

(Spectromètre de Masse par Accélérateur) et nouvelles problématiques », In Situ [En ligne], 9| 2008,

mis en ligne le 18 avril 2012, consulté le 23 avril 2019. URL : http://journals.openedition.org/ insitu/3342 ; DOI : 10.4000/insitu.3342

Ce document a été généré automatiquement le 23 avril 2019.

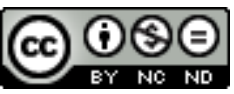

In Situ Revues des patrimoines est mis à disposition selon les termes de la licence Creative Commons Attribution - Pas d'Utilisation Commerciale - Pas de Modification 4.0 International. 


\title{
Le programme ARTEMIS : nouvel outil pour la datation radiocarbone AMS (Spectromètre de Masse par Accélérateur) et nouvelles problématiques
}

\author{
Cyrille Billard
}

1 Le ministère de la culture et de la communication, l'IRSN (Institut de Radioprotection et de Sûreté Nucléaire) et l'IRD (Institut de Recherche pour le Développement ex ORSTOM) disposent aujourd'hui du nouvel équipement ARTEMIS (Accélérateur pour la Recherche en sciences de la Terre, Environnement, Muséologie). Inauguré le 8 avril 2004 au centre du Commissariat à l'Energie Atomique (CEA) de Saclay, le CNRS, le CEA doit permettre d'analyser plus de 4000 échantillons par an.

2 Ce projet ambitieux, initié dès 1998, a pour objectif de répondre quantitativement et qualitativement aux besoins des laboratoires opérant dans le domaine de l'environnement et du climat, mais aussi de la communauté archéologique et muséographique.

3 Le nouvel appareil devrait répondre à un besoin annuel évalué à 4500 datations radiocarbones. Après quasiment deux années de fonctionnement, on est en mesure de dresser un premier bilan des nouvelles procédures mises en place, mais aussi des nouvelles applications offertes aux chercheurs par cet appareil.

\section{ARTEMIS une machine de nouvelle génération}

L'intuition et les travaux de L. Libby en 1949 concernant la mesure du C14 résiduel - qui lui valurent d'ailleurs le prix Nobel en 1960 - ont été grandement perfectionnés depuis, 
notamment grâce à la technologie SMA (Spectromètre de Masse par Accélérateur), fondée non plus sur la radioactivité d'un échantillon mais sur le comptage des atomes de ${ }^{14} \mathrm{C}$ qu'il contient.

5 Apparue dans les années 1980, cette méthode a autorisé des avancées majeures. Aujourd'hui, les caractéristiques d'ARTEMIS permettent d'améliorer encore les performances de la technologie SMA et autorisent des mesures sur des échantillons d'un poids inférieur au milligramme de carbone au lieu de plusieurs grammes et avec une très grande rapidité d'exécution (fig. $\mathbf{n}^{\circ} \mathbf{1}$ ). Actuellement les temps de comptage pour un appareil de technologie SMA sont évalués à moins d'une heure au lieu de plusieurs jours, voire plusieurs semaines, sur des machines conventionnelles.

Figure 1

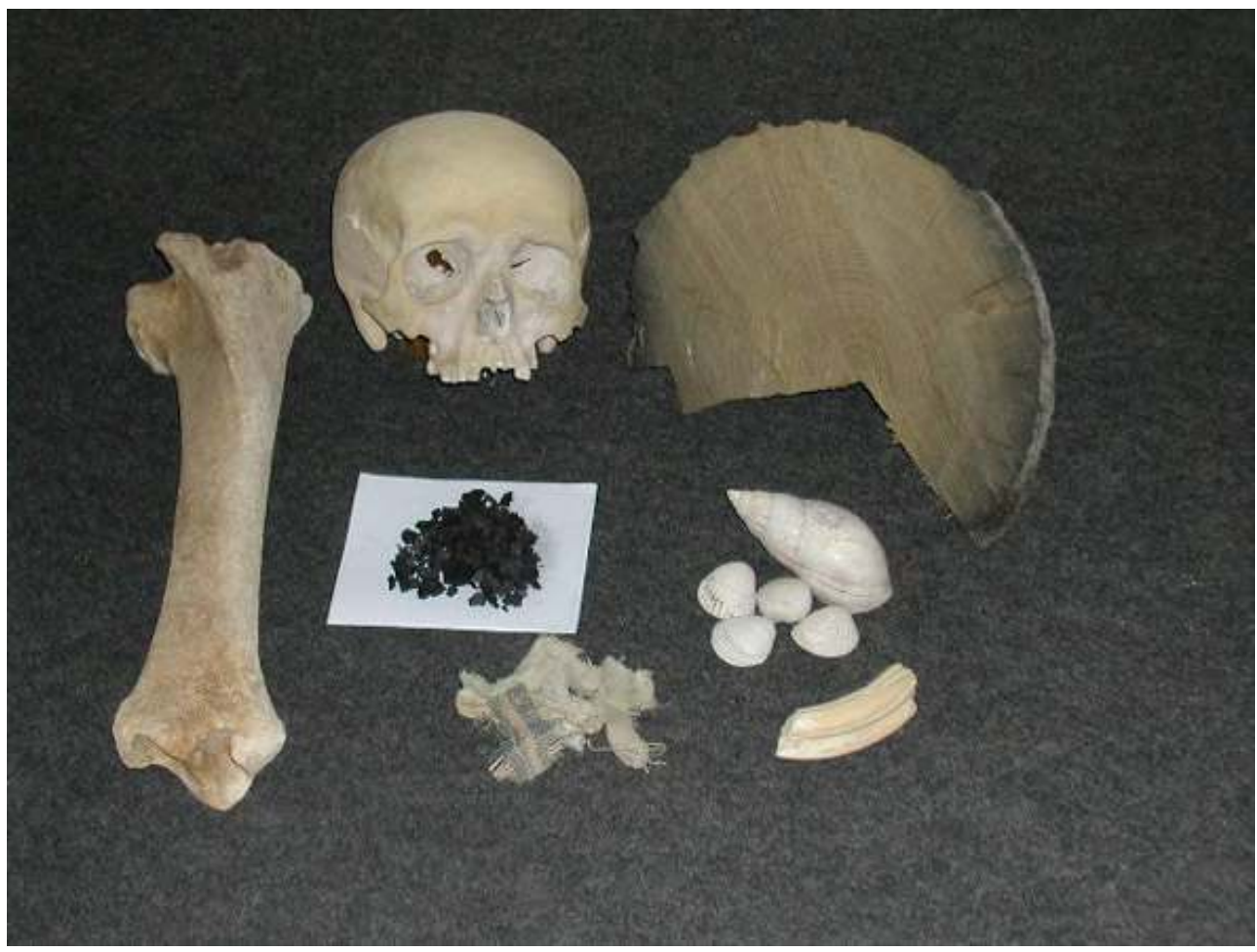

Quelques échantillons datables

(c) Centre de Datation par le Radiocarbone de Lyon

\section{Un important partenariat institutionnel financier et scientifique}

En 2001 une convention permettant l'acquisition en commun de la machine et son installation dans un environnement scientifique et technique performant a été signée entre les cinq partenaires. Le centre national de la recherche scientifique (CNRS), le commissariat à l'énergie atomique (CEA) l'institut de radioprotection et de sûreté nucléaire (IRSN) et l'Institut de recherche pour le développement (IRD) se sont associés pour l'achat de l'équipement ARTEMIS et ont sollicité le ministère de la culture et de la communication (Mission de la recherche et de la technologie, MRT), au même titre que l'INSU et le département des sciences chimiques du CNRS, pour contribuer à 
l'investissement à hauteur de 0,255 M€ HT (fig. $\mathbf{n}^{\circ}$ 2). Cet engagement financier important a décidé de la participation, déterminante, du Conseil régional d'Ile de France par une subvention de 0, $45 \mathrm{M€}$.

Figure 2

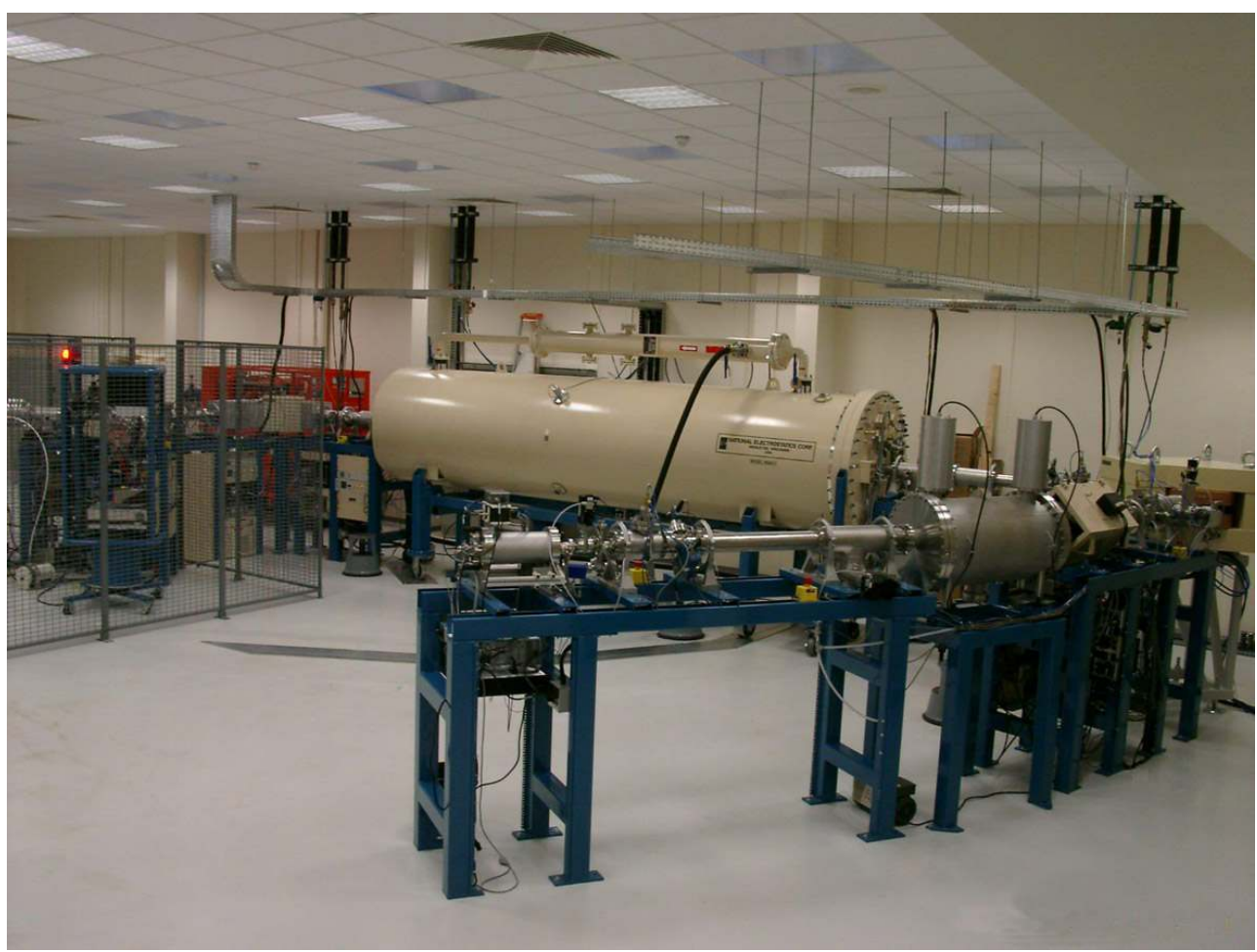

Spectromètre de masse couplé à un accélérateur : « ARTEMIS »CEA/Saclay

Cottereau, Evelyne (c) LMC14-UMS 2572 CEA-Saclay, 2003

Les moyens humains et matériels sont aujourd'hui regroupés au sein d'une Unité Mixte de Service et de Recherche (LMC14), majoritairement implantée à Saclay.

La mise en œuvre des orientations scientifiques du LMC14 est définie par l'ensemble des partenaires au sein d'un Comité de direction siégeant ordinairement une fois par an. La tâche du LMC14 est de gérer le fonctionnement, le développement de l'équipement ainsi que sa maintenance. Par son investissement initial et la participation de certains de ses agents au fonctionnement de la structure, le ministère de la culture détient des droits d'accès représentant environ $7 \%$ des analyses.

9 Les secteurs de l'archéologie et des musées réalisent la préparation de leurs échantillons en s'appuyant d'une part sur les capacités du Centre de recherche et de restauration des musées de France (C2RMF) pour la direction des musées de France et d'autre part sur celles du Centre du Radiocarbone de Lyon (UMR conventionnée 5138 « Archéométrie et archéologie ») pour le ministère de la culture, mais également pour le CNRS/SHS. Cette dernière organisation à conduit les partenaires à développer une collaboration plus spécifique. 


\section{Nouvelles procédures de programmation}

10 En effet, il devient nécessaire de coordonner les demandes des principales disciplines consommatrices d'analyses ${ }^{14} \mathrm{C}$, dans le cadre d'une programmation scientifique équilibrée et optimisée en matière de datation.

\section{Le partenariat CNRS/MCC}

$11 \mathrm{Au}$ total, ce sont 460 droits aux analyses qui existent désormais pour la Sous-direction de l'archéologie, de l'ethnologie, de l'inventaire et du système d'information (Sdarchetis) et 350 droits pour la Direction des musées de France/C2RMF, totalement dédiés à la seule mesure d'âge SMA et dont la programmation devra transiter par les comités d'experts propres au ministère de la culture.

12 Le SHS du CNRS propose, quant à lui, une offre directe de 700 dates SMA dans son réseau d'unités de recherche.

\section{Conseils scientifiques de programmation}

13 En 2006, un comité de programmation des mesures a été installé sur la base d'une représentation croisée des institutions (le département Sciences Humaines et Sociales (SHS) du CNRS, le Ministère de la culture et de la communication, plus récemment l'Institut national de recherches archéologiques préventives (INRAP).

La coordination est assurée par la mission de la recherche et de la technologie (MRT) à l'échelon central du ministère de la culture et de la communication. Elle intègre l'ensemble des services déconcentrés de la DAPA. Outre le domaine d'intervention des SRA, les CRMH et les SDAP ont en effet la possibilité de réaliser des études sur certains monuments (charpentes, bois d'œuvre, mortiers) et sur des objets manufacturés à caractère historique. Au sein de la Direction des Musées de France, les conseillers musées en DRAC et les conservateurs de musées sont également susceptibles de programmer des études sur les séries muséographiques (par exemple, objets mobiliers du Paléolithique supérieur). La Direction des Musées de France (DMF) (Centre de recherche et de restauration des musées de France, C2RMF) et la DAPA collaborent à la datation directe des œuvres pariétales.

Parallèlement, le département SHS a créé son propre comité dans lequel sont accueillis deux experts du ministère de la Culture afin de favoriser la circulation de l'information relative aux dossiers communs.

Cet échelon commun a pour tâche de garantir la traçabilité de chaque échantillon passant par la filière « archéologie-ARTEMIS » ainsi qu'une programmation cohérente. Il est aussi le garant du retour de l'information vers l'ensemble des services déconcentrés et leurs partenaires. Enfin, il a pour ambition de dégager une vision nationale en matière de datation numérique et d'organiser la ressource en vue d'élaborer des programmations effectives et harmonisées. 


\section{Mise en place de la programmation nationale}

17 Un volant de 460 droits-machine est théoriquement disponible annuellement dans le cadre de la Sous-direction de l'archéologie, de l'ethnologie, de l'inventaire et du système d'information. Sur cette base, la sous-direction et le département SHS du CNRS sont convenus d'une collaboration étroite autour d'une politique de recherche coordonnée incluant notamment le regroupement de tous les résultats des mesures au sein d'une base de données informatique commune: BANADORA: «BAnque NAtionale de DOnnées RAdiocarbone pour l'Europe et le Proche Orient » instituée depuis plusieurs années à l'initiative du Centre de datation par le radiocarbone (CDRC) de Lyon.

\section{Les conditions d'une programmation sur ARTEMIS : une nouvelle donne}

Auparavant, la réalisation d'une datation SMA par un laboratoire pouvait être assimilée à une prestation de service facturée aux chercheurs. Elle s'intègre dorénavant dans une programmation nationale. Soumettre aujourd'hui une demande dans le cadre d'un projet de datations SMA implique, de la part du demandeur, le respect de plusieurs conditions. D'une part, ARTEMIS n'assure que des mesures d'âge radiocarbone par accélérateur, impérativement réservées à des échantillons qui ne pourraient être datés par la méthode classique, moins coûteuse. D'autre part, la demande doit s'inscrire dans les limites des moyens et dans le cadre de la programmation scientifique de chaque service régional de l'archéologie (SRA). Il est effectivement prévu que chacun assure le libre usage de son contingent déconcentré, mais une politique incitative pourra être mise en œuvre au sein des UMR conventionnées par la mise en commun de droits machines du MCC et du CNRS autour de thèmes de recherche prioritaires.

\section{La procédure de soumission}


Figure 3

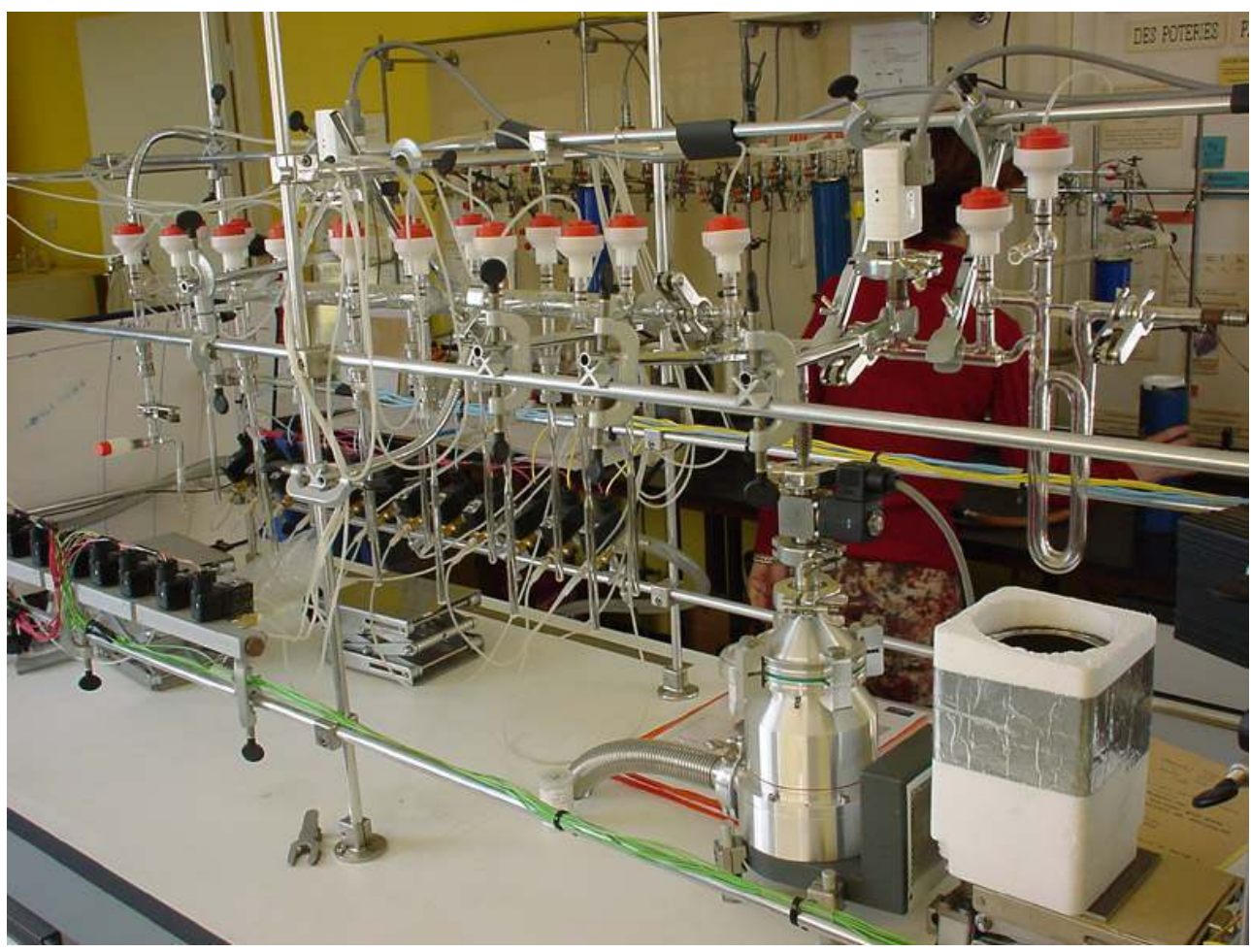

Banc de préparation automatique pour les échantillons datés par Accélérateur

(c) Centre de Datation par le Radiocarbone de Lyon

Depuis le 1er février 2007 une fiche électronique de renseignement laboratoire, désormais commune au CNRS/SHS et au ministère de la culture, dédiée à la programmation sur ARTEMIS, est en service sur le site du laboratoire de radiocarbone à l'adresse suivante (fig. $\mathbf{n}^{\circ}$ 3) :

http://www.archeometrie.mom.fr/artemis/

21 La procédure de soumission est détaillée dans un Guide d'utilisation du programme ARTEMIS, élaboré par la direction de l'Architecture et du patrimoine.

Le demandeur adresse directement sur cette fiche les informations qui sont ensuite traitées en préalable par le laboratoire de mesure du radiocarbone. Ainsi les demandes peuvent être évaluées techniquement sur la base de leur potentiel de «faisabilité » sous technologie SMA, puis triées et retransmises aux service régionaux de l'archéologie concernés sous forme de fichiers de tableurs reprenant l'ensemble des rubriques éventuellement assorties des commentaires techniques appropriés. Ce premier filtre a pour objectif principal de soulager les services régionaux de l'archéologie de la complexe évaluation technique de faisabilité d'une datation. Il appartient ensuite à chaque service de construire, sur la base de ses choix initiaux, sa programmation annuelle au prorata du nombre de droits alloués pour l'année. Il lui revient aussi de recueillir, le plus largement possible, l'avis des instances d'évaluation que sont les Commissions interrégionales de la recherche archéologique (CIRA). Les demandes pourront alors être transmises au comité ARTEMIS/SDarchetis directement sous format de tableur, accompagnées de l'argumentaire du service régional de l'archéologie, et complétées des informations utiles sur les sites, les opérations et les validations scientifiques. Enfin, il incombe également 
aux services régionaux de fournir les informations à même de permettre la mise en relation avec PATRIARCHE, base nationale de données archéologiques.

Après attribution du numéro d'ordre, indispensable au traitement des échantillons par les laboratoires, le comité de programmation SDArchétis informe conjointement les services régionaux de l'archéologie et le laboratoire afin que le demandeur puisse envoyer ses échantillons pour traitement dans le courant du mois suivant. Les échantillons sont donc adressés au CDRC de Lyon par le demandeur. Dans le cas de certains échantillons, une expertise préalable du CDRC de Lyon est parfois nécessaire, en particulier pour les échantillons d'os mal conservés qui sont susceptibles de ne pas livrer suffisamment de collagène.

\section{Nouvelles applications}

La réflexion et la définition de grands axes thématiques en matière de datation absolue n'est pas un sujet nouveau en sciences humaines et a occupé depuis plusieurs années l'ensemble de la communauté dans les domaines de l'histoire, de l'archéologie et du patrimoine. La journée de réflexion méthodologique organisée en mars 2002 conjointement avec le ministère de la recherche (SHS du CNRS) et le ministère de la culture (Mission de la recherche et de la technologie (MRT)- Direction de l'architecture et du patrimoine (DAPA)/SDarchetis et DMF/C2RMF) et dont les actes ${ }^{1}$ ont été publiés par le C2RMF en est un témoignage parmi d'autres.

De plus, au terme de deux années de fonctionnement d'ARTEMIS, les chercheurs ont exprimé leurs besoins. Commencent ainsi à se dessiner quelques grandes thématiques que l'outil ARTEMIS contribuera fortement à développer.

\section{Datation d'objets ethnographiques ou artistiques}

L'utilisation de la technologie radiocarbone AMS pour dater des objets de musée s'inscrit pleinement dans la mission scientifique de ces établissements, que l'on prenne en compte des éléments d'art mobilier osseux préhistorique, des textiles anciens, ou des tableaux primitifs flamands.

\section{Préhistoire et grottes ornées}

Dans le domaine de l'archéologie, les enjeux en matière de datation numérique sont nombreux et déterminants car mesurer la teneur en $14 \mathrm{C}$, de matériaux archéologiques permet d'apprécier des âges pouvant remonter jusqu'à 35000 ans en routine et 40 voire 50000 ans de manière expérimentale. La mesure $\mathrm{du}{ }^{14} \mathrm{C}$ a permis de dater les plus anciennes manifestations artistiques pariétales de l'histoire humaine dans les grottes ornées. Plus globalement, elle permet d'envisager la constitution de nouveaux référentiels chronologiques, calibrés et exprimés en années solaires, par l'actualisation des corpus de datation, autorisant le découpage des grandes périodes chronologiques ou culturelles.

\section{L'architecture médiévale}

Depuis une vingtaine d'années, le C14 est de plus en plus utilisé pour les périodes historiques. Dans les années 1960, des tentatives de datation de mortiers de chaux se sont 
malheureusement heurtées à plusieurs sources d'erreur. Dès lors, l'analyse du C14 sur les charbons de bois inclus dans les mortiers constitue la principale méthode (avec la thermoluminescence et l'archéomagnétisme sur les terres cuites architecturées) pour dater des bâtiments en élévation, en particulier lorsque les niveaux d'occupation ont disparu ou ne sont pas accessibles, comme dans le cas de bon nombre de monuments historiques (exemples récents de l'abbaye de Saint-Germain-d'Auxerre, du prieuré SaintSauveur-de-Melun (Seine-et-Marne) ou de l'église Notre-Dame-sous-Terre sur le MontSaint-Michel).

\section{L’archéologie funéraire}

L'archéologie funéraire a connu des progrès considérables en l'espace de quelques décennies. Pourtant, jusqu'à présent, les datations sur un même ensemble funéraire étaient peu nombreuses. Elles se sont particulièrement développées dans le domaine de l'archéologie des cimetières médiévaux. Les approches les plus récentes, $\mathrm{ADN}$, caractères discrets, ont conduit les chercheurs à travailler davantage sur le temps court, sur l'événementiel. On analyse les possibles regroupements familiaux ou les regroupements en fonction du statut social. L'analyse du geste funéraire ou des phénomènes taphonomiques se situent également dans le temps court.

Dans le meilleur des cas, en fonction des moyens alloués, on cherchait à dater une phase de dépôt funéraire ou un type de tombe au sein d'un site. Le programme ARTEMIS se place complètement dans cette nouvelle perspective en permettant d'envisager de multiplier les datations des individus d'un grand ensemble funéraire.

\section{Le bois et les datations AMS}

31 A priori, le bois est le matériau qui se prête le mieux à des datations par la méthode classique. Toutefois, le problème est que, dans ce cas, l'échantillon va porter sur 15 à 20 cernes, tandis que la technologie par accélérateur permet la datation d'un unique cerne.

De nombreuses régions présentent une absence de courbe de référence dendrochronologique pour certaines périodes : dans l'ouest ou dans le sud de la France en particulier. Plusieurs d'entre elles ont le projet de se lancer dans l'élaboration de tels référentiels qui nécessitent de mener une politique de prélèvements tous azimuts (bois gorgés d'eau dans les tourbes, charpentes, bois archéologiques...).

En multipliant les datations AMS sur un même bois, la méthode du wiggle-matching (qui peut se traduire par "mise en concordance des oscillations») permet aujourd'hui d'affiner la datation des échantillons tout particulièrement pour des zones géographiques sans référentiel dendrochronologique.

34 Même en gardant à l'esprit des exigences comptables (la datation C14 des bois par la méthode classique est en principe moins coûteuse), il est clair que l'outil ARTEMIS apportera beaucoup à la dendrochronologie et à l'étude des sites en milieux humides. L'établissement de référentiels dendrochronologiques interrégionaux est l'exemple type de programme pouvant bénéficier d'une mise en commun des moyens dont disposent les UMR et les SRA. 


\section{Le paléoenvironnement}

L'approche de la chronologie dans les études paléoenvironnementales se place encore trop souvent dans le temps long, celui des sciences de la terre. Or la prise en compte de l'impact anthropique et des mutations rapides que connaissent les paysages exigent l'élaboration de chronologies de plus en plus fines. La possibilité de multiplier les datations sur une même colonne stratigraphique ouvre de nouvelles perspectives dans ce sens. La technique AMS permet également de prendre en compte des phénomènes d'érosion au travers d'échantillons contenus dans des sédiments peu ou pas organiques (microcharbons de bois liés à des brûlis par exemple).

\section{L'archéologie de la zone intertropicale humide}

Lors des deux premières années de fonctionnement du programme ARTEMIS, les départements d'Outremer, auxquels est venue s'ajouter la Polynésie française, ont manifesté un vif intérêt pour ce dispositif. Cet intérêt provient d'une conjonction de facteurs: un retard évident dans l'élaboration des chronologies, une mauvaise conservation et la petite taille des charbons de bois (en particulier dans les zones insulaires), ainsi que les difficultés rencontrées dans la datation des coquillages pour cause de mauvaise évaluation de l'effet réservoir.

Les chercheurs disposent donc d'un outil patrimonial remarquable, qui nécessite un changement d'attitude de leur part et dont il convient de ne pas banaliser les performances. Alors que la réalisation d'une datation était jusqu'à présent trop souvent assimilée à une simple prestation, la gestion de cet outil doit permettre d'initier une programmation concertée des datations, bien intégrée aux grandes problématiques. Enfin, cette nouvelle mission de programmation, dévolue en particulier aux services régionaux de l'archéologie, exige une connaissance suffisante de la technologie SMA pour être à même d'apporter une analyse critique des demandes.

\section{NOTES}

1. Archéologie, patrimoine culturel et datation parle carbone 14 par spectrométrie de masse par accélérateur. Table-ronde du 22 mars 2002. Paris, C2RMF. 


\section{RÉSUMÉS}

La mise en route du nouvel équipement ARTEMIS (Accélérateur pour la Recherche en sciences de la Terre, Environnement, Muséologie, Implanté à Saclay) à partir de 2004 ouvre de nouvelles perspectives scientifiques et conduit à de nouvelles procédures de soumission des échantillons destinés à une datation ${ }^{14} \mathrm{C}$. Le MCC dispose aujourd'hui de droits alloués aux services régionaux de l'archéologie, services du ministère de la Culture, confrontés à ce type de demandes (musées, monuments historiques). Il impose désormais une nouvelle démarche de programmation scientifique des datations, associant une analyse critique des demandes.

Since 2004, the availability at Saclay of a new ARTEMIS installation (Accélérateur pour la Recherche en sciences de la Terre, Environnement, Muséologie) has opened new scientific perspectives and led to new procedures for submitting samples for carbon-14 dating. The French Ministry of Culture has the possibility of using this tool for radiocarbon dating at the request of its regional archaeological services or other services, such as museums and the historic monuments administration. This use now implies a new approach to the scientific planning for dating problems, associated with a critical analysis of the requests.

\section{INDEX}

Mots-clés : accélérateur, archéologie, archéologie funéraire, architecture médiévale, ARTEMIS, BANADORA, C2RMF, carbone 14, CNRS, Commissariat à l'Energie Atomique, datation, échantillons, grottes ornées, INRAP, Institut de Radioprotection et de Sûreté Nucléaire, Institut de Recherche pour le Développement, ORSTOM, paléoenvironnement, préhistoire, procédure de soumission, bois

Keywords : accelerator mass, carbon-14, radiometric dating, spectrometry

\section{AUTEUR}

\section{CYRILLE BILLARD}

Conservateur du patrimoine. Direction régionale des affaires culturelles de Basse-Normandie, service régional de l'archéologie. cyrille.billard@culture.gouv.fr 